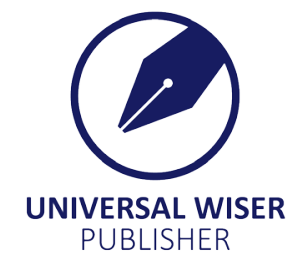

\title{
Modelling Colloidal Stability in Gold Nanoparticles Synthesis - A Review
}

\author{
Emmanuel Agunloye, Faith U. Babalola* \\ Department of Chemical and Petroleum Engineering, University of Lagos, Akoka, Lagos, Nigeria \\ E-mail: Fu_babalola@yahoo.com, fubabalola@unilag.edu.ng
}

\begin{abstract}
Nanoparticles are commonly synthesized in colloidal systems, a liquid with suspended macromolecules. By the Brownian motion, these particles can collide and aggregate, leading to larger particles. To stabilize the aggregation process, charges induced by ions cause repulsion among the particles. Through the works of researchers in the past, various models for describing particles aggregation and stabilization have been developed and modified. These models are based on the popular DLVO theory, named after Derjaguin, Landau, Verwey and Overbreek. In this work, evidence illustrating aggregation and stabilization in gold nanoparticles synthesis is reported. Thereafter, models for describing aggregation by the Brownian motion and stabilization by the electrostatic effect are reviewed. The electrostatic effect among the particles is mathematically expressed as the cumulative sum of the Van der Waal's energy of interaction and the electrostatic energy of repulsion. As the resulting stabilization model is too complex to solve, past researchers reported a simplified stabilization submodel and employed it in describing gold nanoparticles synthesis. Unfortunately, as shown in this review, the submodel failed to describe the synthesis as the aggregation process never stopped, thus making a case for a new modelling approach.
\end{abstract}

Key Words: gold nanoparticles, citrate synthesis, aggregation, population balance modeling

\section{Introduction}

Nanotechnology is a technology that explores materials with sizes in the range 1-100 $\mathrm{nm}$ for various applications ${ }^{[1]}$. It involves the synthesis of these materials, called nanoparticles (NPs), their modification (or functionalization) and finally their application. Today, various consumer products come from nanotechnology. To meet these growing applications, NPs can be synthesized by different methods, classified as top-down or bottom-up approaches ${ }^{[1]}$. In the top-down approach for gold nanoparticles synthesis, bulk gold can be ground in a ball mill; then, the resulting particles are air-classified to recover NPs. In the bottom-up approach, nanoparticles are synthesized from sub-size particles such as atoms and molecules. Many gas and liquid-phase synthesis methods fall under the bottom-up approach. Liquid-phase methods, which are simple and cheap, offer a one-stop panacea to the synthesis of NPs in general and gold nanoparticles (GNPs) in particular. They can produce nanoparticles of sizes $1-200 \mathrm{~nm}$, which can be easily functionalised for potential applications. These methods involve many techniques in tuning the nanoparticles size, form and geometry. These many techniques are however mostly carried out in colloidal systems.

A colloidal system is made-up of macromolecules, such as polymers, dispersed in a liquid such as water. Because of their chemical nature, these polymers do not dissolve in water, thereby forming a heterogeneous solution. However, to an unaided eye, the solution appears homogeneous. Hence, colloidal systems are also called micro-heterogeneous systems. Advantages of using them in the synthesis of nanoparticles include safety, cost effectiveness and low energy requirement. In nanoparticles synthesis, the polymers are referred to as surfactants. These form cavities in which nanoparticles can form. They can assume various arrangements, perform many functions, and be consequently used in diverse applications in nanotechnology.

Although decreasing particle size should increase reactivity, NPs synthesized in a colloidal system are stabilized by cavities of surfactants that suppress size increase and reactivity of NPs. Subsequently, these NPs based on their properties such as composition, size and shape find various applications. For example, GNPs are one of the most applied nanoparticles ${ }^{[2]}$; they are used in biomedicine, catalysis, and optics ${ }^{[3]}$. In a widely used synthesis method of gold nanoparticles, called the citrate synthesis method, sodium citrate reduces tetrachloroauric acid. Apart from reducing the precursor, the citrate

Copyright (C2020 Faith U. Babalola, et al.

DOI: https://doi.org/10.37256/nat.122020174.59-69

This is an open-access article distributed under a CC BY license

(Creative Commons Attribution 4.0 International License)

https://creativecommons.org/licenses/by/4.0/ 
also stabilizes the nanoparticles and regulates the $\mathrm{pH}$ of the reaction mixture, which is an important factor in the synthesis. The gold in the precursor evolves by processes such as reactions, nucleation, aggregation and growth. Reactions involve changing the chemical nature of the substances such as reducing the precursor to gold while nucleation is the generation of the smallest particles from the aqueous phase. On the other hand, aggregation is the coming together of smaller particles to form larger particles and growth is the increase in particle size due to the addition of molecules to the characteristic arrangement of the particle.

Stability of the synthesis depends on whether every process becomes insignificant with time. For reactions, nucleation and growth, reactants in the aqueous phase such as $\mathrm{AuCl}_{4}^{-}, \mathrm{AuCl}_{3}(\mathrm{OH})^{-}, \mathrm{CtH}_{2}^{-}, \mathrm{CtH}^{2-} \mathrm{H}^{+}$and $\mathrm{OH}^{-}$convert to either products in the aqueous phase or gold in the particle phase ${ }^{[3]}$. For example, in the nucleation step, which requires $\mathrm{AuCl}_{4}^{-}$and $\mathrm{CtH}^{2-}$ to occur, depleting any of the reactants' concentration would lead to the completion of the step. Therefore, in the citrate synthesis conducted in a batch reactor by Wuithschick et al ${ }^{[2]}$, each of the reactions, nucleation and growth would stop as the concentration of the limiting reactant becomes insignificant. Aggregation, on the other hand, occurs in the presence of particles, which are always available in a nanoparticles synthesis mixture. By the Brownian motion, caused by the thermal energy, these nanoparticles agitate, collide and can aggregate. However, the aggregation process does not continue indefinitely. For example, in the citrate synthesis method, Wuithschick et al. ${ }^{[2]}$ reported the formation of seed particles, after which aggregation is insignificant in the synthesis. As experimental data have confirmed particles aggregating, and thereafter stabilizing, the citrate synthesis method is the focus of this review paper.

Models for describing the aggregation process are reported in literature ${ }^{[4-5]}$. To describe the stabilization of nanoparticles to aggregation, the aggregation model for particles of sizes smaller than $1 \mathrm{um}$ was modified to include a stability factor, which is a function of the interaction energy among particles ${ }^{[5-6]}$. For metal nanoparticles, the interaction energy due to the forces of attraction and repulsion is required to determine colloidal stability. For particle-particle interaction, models for these forces and their resulting interaction energy have been described in the popular DLVO theory, reported by Derjaguin and Landau ${ }^{[7]}$ and Verwey and Overbreek ${ }^{[8]}$. In this theory, the total interaction energy between two particles is the sum of the Van der Waal's energy of attraction and electrostatic energy of repulsion. Based on this theory, an expression was developed for the stability of identical particles against aggregation in terms of the total interaction energy between a pair over the whole space of interaction. This interaction energy has a peak value at a separation between the particles equivalent to the electric double layer. Although incorporating DLVO models into the aggregation model should ensure colloidal stability, it yields a complex mathematical expression.

In the past, several authors have reported simplified expressions for the aggregation process. Recently, Agunloye et al. ${ }^{[3]}$ reported empirical relations for finding the seed particles size, a size corresponding to colloidal stability. However, the size was not related to the interaction energy and the empirical relations cannot be used in the aggregation model. Earlier on, Reerink and Overbreek ${ }^{[9]}$ mathematically simplified the complex aggregation model to a form in terms of the concentration of ions. Thereafter, Kumar et al. ${ }^{[10]}$ developed a model for the formation of GNPs in citrate synthesis method and employed the simplified aggregation model in describing the aggregation process. Unfortunately, as alluded by Agunloye et al. ${ }^{[11]}$, the model by Kumar et al. ${ }^{[10]}$, in general, failed to describe the citrate synthesis and in particular, the aggregation sub-model did not illustrate colloidal stability.

In the citrate synthesis method, Chow and Zukoski ${ }^{[12]}$ reported a graph of the ratios of the total interaction energy to the thermal energy increasing with the surface potential. Therefore, as the surface potential increases, particles would become more stabilized against aggregation. The derivation of their simplified aggregation model for the graph needs to be reviewed.

This work therefore reviews the aggregation model, the stabilization model by electrostatic effect, the particle-particle interaction model and the simplified aggregation model. Before reviewing these models, this work briefly discusses the experimental investigation of Wuithschick et al. ${ }^{[2]}$, to illustrate aggregation and stabilization of nanoparticles in the citrate synthesis method.

\section{Methodology}

\subsection{Citrate synthesis method}

The citrate synthesis method is commonly used to produce GNPs by reacting tetrachloroauric acid with sodium citrate in an aqueous solution ${ }^{[10,13]}$. In this synthesis method, the precursor is tetrachloroauric acid, the reducing agent is sodium citrate, and the solvent is water. Turkevich et al. ${ }^{[14]}$ used techniques such as the electron microscopy to investigate the synthesis in a batch reactor under different process conditions (i.e. the synthesis temperatures and 
reactants' concentrations). In their standard synthesis condition, where the synthesis temperature was $100{ }^{\circ} \mathrm{C}$ and the initial concentrations of tetrachloroauric acid and sodium citrate were 0.255 and $1.938 \mathrm{~mol} / \mathrm{m}^{3}$, respectively, the authors reported in the final mixture spherical GNPs of a mean size of $20 \mathrm{~nm}$.

In the past, researchers explained the GNPs synthesis through the pioneering work of Turkevich et al. ${ }^{[14]}$, which suggested that the particles form via a nucleation-growth mechanism. According to this mechanistic description of the synthesis, also known as the Turkevich organizer theory, tetrachloroauric acid reacts with sodium citrate to form gold chloride and dicarboxy acetone (DCA). Subsequently, the latter (which acts as nucleation agent) catalyses the conversion of gold chloride to gold nuclei, while decomposing into acetone. Its decomposition eventually prevents the generation of new nuclei (nucleation stops), leaving behind unconsumed gold chloride that reacts on the surface of the particles thereby making them grow. Nucleation and growth are therefore decoupled.

While the Turkevich organizer theory did not report aggregation, the seed-mediated mechanism involves nucleation, aggregation and growth in the particle phase. Aggregation in the citrate synthesis method was first reported by Chow and Zukoski ${ }^{[12]}$ while investigating the synthesis with a combination of the UV-Vis spectroscopy and electron microscopy. Using this set-up as well, Ji et al. ${ }^{[15]}$ reported a more extensive study, which first pointed out the role of $\mathrm{pH}$ and illustrated how it affects the effect of aggregation in the synthesis. In addition to this set-up, Polte et al. ${ }^{[16]}$ and Wuithchick et al. ${ }^{[2]}$ used a combination of X-ray absorption near edge structure and Small-angle X-ray scattering (SAXS) techniques to investigate the synthesis. This equipment provides time-resolved in situ information on the size of nanoparticles of about $2 \mathrm{~nm}$ or larger and of their number concentration, offering a reliable account of the synthesis. Figure 1 shows the SAXS evidence for the presence of aggregation in the synthesis.

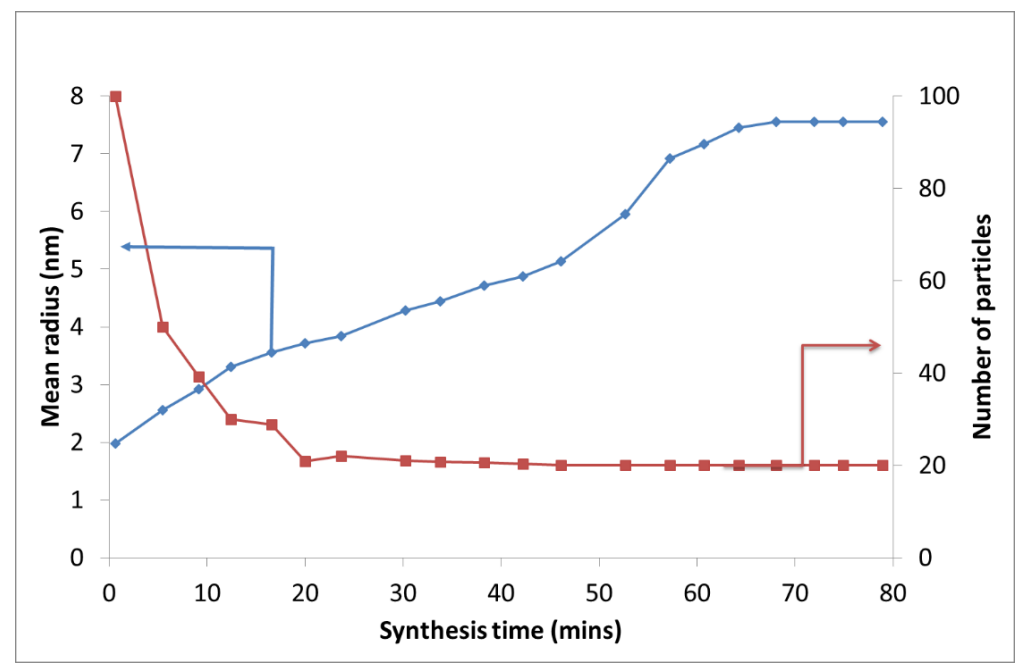

Figure 1. Evolution of the mean radius and of the number of particles in the citrate synthesis method. The values of the initial concentrations of $\mathrm{HAuCl}^{4}$ and $\mathrm{Na}_{3} \mathrm{Ct}$ in the synthesis solution are $0.25 \mathrm{~mol} / \mathrm{m}^{3}$ and $2.5 \mathrm{~mol} / \mathrm{m}^{3}$, respectively. The mixture temperature is $75{ }^{0} \mathrm{C}$. Data reproduced from Figure 2(d) of Polte et al. ${ }^{[16]}$

From Figure 1, the number of particles, which is at the maximum early in the synthesis, declines for about onethird of the synthesis time, and remains constant thereafter. In the process, the size polydispersity (i.e. standard deviation) reduced ${ }^{[16]}$. The maximum number of particles corresponds to the nucleated nanoclusters, which coalesce to form the seed particles, reflected in the decline of the particle number. Later on, the number of particles remains constant while particle size steadily rises. This suggests that particles stop coalescing and start growing.

Stability of the synthesis can be further illustrated by considering mechanisms of stabilization by surfactants. A simple surfactant comprises a functional group head (the polar part) and an alkyl tail (the apolar part). The polar part dissolves in a polar medium while the apolar part dissoves in an apolar medium. This property makes surfactants assume various arrangements such as a film or a micelle of surfactant molecules. Figure 2 shows a typical surfactant (dodecanethiol) and a possible arrangement on a polar surface. 


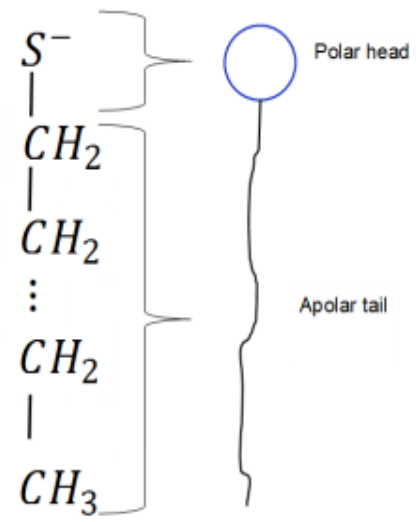

a) Surfactant

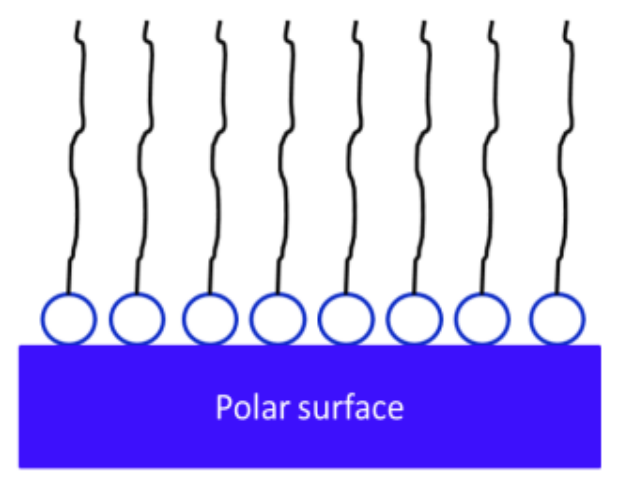

b) Surfactants arrangement on a polar surface

Figure 2. The structure and arrangement of dodecanethiol on a polar surface; a: skeletal structure of dodecanethiol; b: arrangement of dodecanethiol on a polar surface

The arrangement of surfactant molecules can either occur before nucleation of nanoparticles in the reaction medium ${ }^{[1]}$ or in-situ along with nucleation ${ }^{[17]}$.

Before nucleation. The configuration of surfactant molecules is formed before nucleation takes place. The produced molecules of the nanoparticles come together inside the cavity formed by the surfactant molecules and therein nucleate. Surfactants in this case act as an 'organizer', as proposed by Turkevich et al. ${ }^{[14]}$. The nucleus starts growing and can only reach the space available in the cavity of the surfactants, and hence stabilizes (Figure 3). The surfactants do not react in the course of the synthesis. An example is the Brust-Schiffrin method, in which dodecanethiol acts as the surfactant ${ }^{[18]}$.

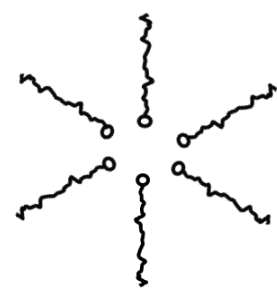

Pre-formed cavity

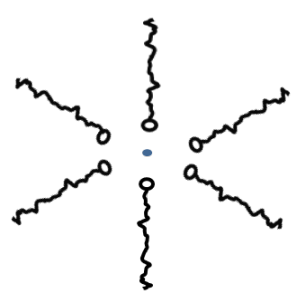

Nucleation

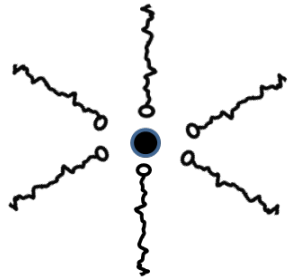

Growth

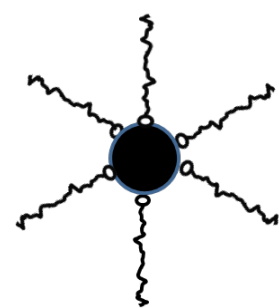

Stabilization

Figure 3. Nucleation, growth, and stabilization of a nanoparticle in a pre-formed surfactant cavity

In-situ. In this case, the nucleus is formed and starts growing while the surfactants remain randomly distributed. After a while, the surfactants reach the surface of the growing nanoparticles, either by adsorption or by complexation, forming a cavity that prevents further growth of the nanoparticles. The latter are therefore stabilized, as shown in Figure 4.

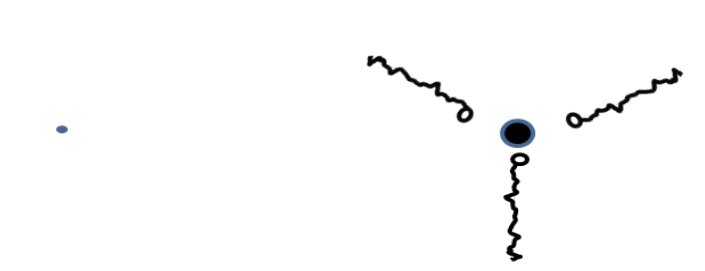

Nucleation

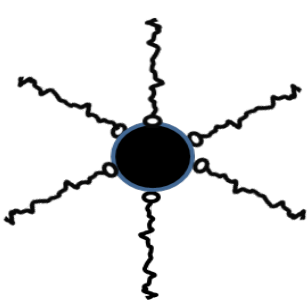

More surfactants form and attach to stabilize the particle

Figure 4. Stabilization of a growing nanoparticle by attachment of surfactant 
In this mechanism, the surfactants are produced in the course of the synthesis. After they are formed, they attach and stabilize the particles. An example is the citrate synthesis method, in which, citrate, a reactant, or any of its derivatives, which is formed in the course of the synthesis, acts as a surfactant, and stabilizes GNPs. This synthesis is the focus of this review paper, as experimental data have confirmed particles aggregating, and stabilizing in the synthesis.

\subsection{Aggregation model}

Particles of sizes below $1 \mu m$ diffuse and exhibit the Brownian motion. Smoluchowski ${ }^{[19]}$ was the first to derive an expression for the aggregation frequency of particles undergoing the Brownian motion. From this derivation, the aggregation kernel between two particles of sizes $\bar{s}$ and $\hat{\boldsymbol{s}}$ is reported as (Hunter ${ }^{[20]}$ ):

$$
\tilde{\omega}_{A}(\bar{s}, \hat{s}, t)=2 \pi\left(D_{\bar{s}}+D_{\hat{s}}\right)(\bar{s}+\hat{s})
$$

From the Stokes-Einstein equation, diffusivity is expressed as (Hunter ${ }^{[20]}$ :

$$
D_{\bar{s}}=\frac{k_{B} T}{3 \pi \overline{\mathrm{s}} \mu}
$$

Where $k_{B}, T$ and $\mu$ are the Boltzmann constant, temperature and fluid viscosity, respectively. Thus, $\tilde{\omega}_{A}(\bar{s}, \hat{s}, t)$ can be expressed as:

$$
\tilde{\omega}_{A}(\bar{s}, \hat{s}, t)=2 \pi\left(D_{\bar{s}}+D_{\hat{s}}\right)(\bar{s}+\hat{s})=\frac{2 k_{B} T}{3 \mu}\left(\frac{1}{\bar{s}}+\frac{1}{\hat{s}}\right)(\bar{s}+\hat{s})
$$

Equation (3) is the well-known Brownian aggregation kernel.

If the aggregating particles are about the same size, then a size-independent kernel $\left(\tilde{\omega}_{A}\right)$ results, so that:

$$
\tilde{\omega}_{A}=8 k_{B} T / 3 \mu
$$

In contrast, particles of disparate sizes have higher rate of aggregation because of the vigorous diffusion of the smaller particle towards its sluggish larger partner. This can be shown using Equation (3). Smoluchowski ${ }^{[19]}$ called Equation (3) the fast aggregation kernel.

\subsection{Stabilization model}

In order to account for the fact that not all collisions are successful at producing a new aggregate, $\tilde{\omega}_{A}$ is divided by a stability factor $W$. This stability factor is a number between 1 and $\infty$. When $W=1$, fast aggregation is obtained; while, when $W=\infty$, no aggregation occurs. There are many factors that can stabilize aggregation. In this work, we limit this to the effect of surface charges and their electric potential.

Surface charges can be positive or negative and can induce either attraction or repulsion between particles depending on the surrounding charges. Let us consider a positively charged GNP. The GNP will attract a layer of tightly-held negative charges around it; this layer, in turn, will attract a second layer of positive charges. These two layers of opposite charges around the initial positively charged GNP are called the electric double layer. This second layer of positive charges is loosely associated with the particle. It is made of free ions that move in the fluid under the influence of electric attraction and thermal motion rather than being firmly anchored. It is thus called the "diffuse layer" ${ }^{[20]}$.

Nanoparticles with surface charges will aggregate only if the cumulative effect of both diffusion and electrostatic interaction brings the nanoparticles together. Fuchs ${ }^{[6]}$ first derived a model for this cumulative effect for monodispersed particles. Subsequently, other authors such as Bogush and Zukoski ${ }^{[21]}$ derived the expression for spherical particles of sizes and. The expression for the aggregation kernel with surface charge is (Bogush and Zukoski ${ }^{[21]}$; Hunter ${ }^{[20]}$ ): 


$$
\frac{\tilde{\omega}_{A}}{W}=\frac{4 \pi\left(D_{\bar{s}}+D_{\hat{s}}\right)}{\int_{(\bar{s}+\hat{s}) / 2}^{\infty} \frac{\exp \left[E_{T} / K_{B} T\right.}{x^{2}} d x}
$$

where $x$ is the distance between the particles, which varies from $(\bar{s}+\hat{s}) / 2$, when the two particles are in contact, to infinity. $E_{T}$ is the total interaction energy, taken to be the sum of the energy due to the Van der Waal's force of attraction and that due to the charge repulsion ${ }^{[20]}$.

As $E_{T}$ increases, $\tilde{\omega}_{A} / w$ decreases, therefore Equation (5) represents the slow aggregation while Equation (3) represents the fast aggregation. Substituting Equation (1) into Equation (5) yields the expression for $W$ as:

$$
W=\frac{(\bar{s}+\hat{s})}{2} \int_{(\bar{s}+\hat{s}) / 2}^{\infty} \frac{\exp \left[{ }^{E T} / k_{B} T\right]}{x^{2}} d x
$$

Equation (6) is the expression for the stabilization of aggregation by electrostatic effect.

\subsection{Particle-particle electrostatic interaction model}

The interaction of colloidal particles due to the forces of attraction and repulsion is described in the popular DLVO theory, named after Derjaguin, Landau, Verwey and Overbreek ${ }^{[7-8]}$. In this theory, the total interaction energy $E_{T}$ between two particles is the sum of the Van der Waals energy of attraction and electrostatic energy of repulsion. The expression for $E_{T}$ is written as:

$$
E_{T}=E_{A}+E_{R}
$$

For spherical particles of diameters $\bar{s}$ and $\widehat{s}$, the expression for $E_{A}$ is (Polte ${ }^{[22]}$ ):

$$
E_{A}=-\frac{\pi^{2} \rho e 1 \rho e 2}{6} Q\left[\frac{2 \overrightarrow{s s}}{4 q^{2}-(\bar{s}+\hat{s})^{2}}+\frac{2 \overrightarrow{s s}}{4 q^{2}-(\bar{s}-\hat{s})^{2}}+\operatorname{In}\left(\frac{4 q^{2}-(\bar{s}+\hat{s})^{2}}{4 q^{2}-(\bar{s}-\hat{s})^{2}}\right)\right]
$$

W where $q$ is the centre to centre distance between the two particles; $Q$ is a parameter that depends on the physical system; $\rho e 1$ and $\rho e 2$ are the electron densities due to the ions attached to the particles of particle-volumes $v p 1$ and $v p 2$, respectively.

While the Van der Waals' energy of attraction brings about aggregation of nanoparticles, the electrostatic energy of repulsion stabilizes them. As described earlier, two layers of opposite charges form around nanoparticles in ionic solutions (the electric double layer). Because of the electric double layer, nanoparticles repel one another. To describe this repulsion, DLVO used the Stern-Gouy-Chapman theory. In this theory, two particles distance $D$ apart carry equal negative charges. The surroundings, on the other hand, contain positively-charged ions, called counterions. The interaction energy for charged surfaces should account for the surfaces and their separation. According to Israelachvili ${ }^{[23]}$, the corresponding repulsion energy is given as:

$$
E_{R}=\int_{D}^{\infty} F_{R} d x=\frac{\pi \bar{s} \hat{s}}{\bar{s}+\hat{s}} \frac{z^{2} e^{2} \Psi o^{2} \rho o}{k_{B} T k^{2}} \exp (-k D)
$$

Equations (8) and (9) can then be substituted in Equation (7) for the total interaction energy for two spherical particles of sizes $\overline{\boldsymbol{S}}$ and $\hat{\boldsymbol{s}}, x$ distance apart as: 


$$
E_{T}=-\frac{\pi^{2} \rho e 1 \rho e 2}{6} Q\left[\frac{2 \overrightarrow{s s}}{4 q^{2}-(\bar{s}+\hat{s})^{2}}+\frac{2 \overrightarrow{s s}}{4 q^{2}-(\bar{s}-\hat{s})^{2}}+\operatorname{In}\left(\frac{4 q^{2}-(\bar{s}+\hat{s})^{2}}{4 q^{2}-(\bar{s}-\hat{s})^{2}}\right)\right]+\frac{\pi \bar{s} \hat{s}}{\bar{s}+\hat{s}} \frac{z^{2} e^{2} \Psi o^{2} \rho o}{k_{B} T k^{2}} \exp (-k x)
$$

This type of expression of $E_{T}$ allows calculating $W$ using Equation (6). For other geometries of interacting charged particles, the expression for $E_{T}$ is different. However, these different expressions yield a similar profile for $E_{T}$. Figure 5 shows this typical profile as the green curve; the blue and red curves show the profiles for $E_{A}$ and $E_{R}$, respectively. The $E_{T}$ curve characteristically has a maximum point, called the energy barrier. Based on this, some past authors simplified the expression of $E_{T}$ and then of $W$. We review their approach to simplifying these expressions in the following section.

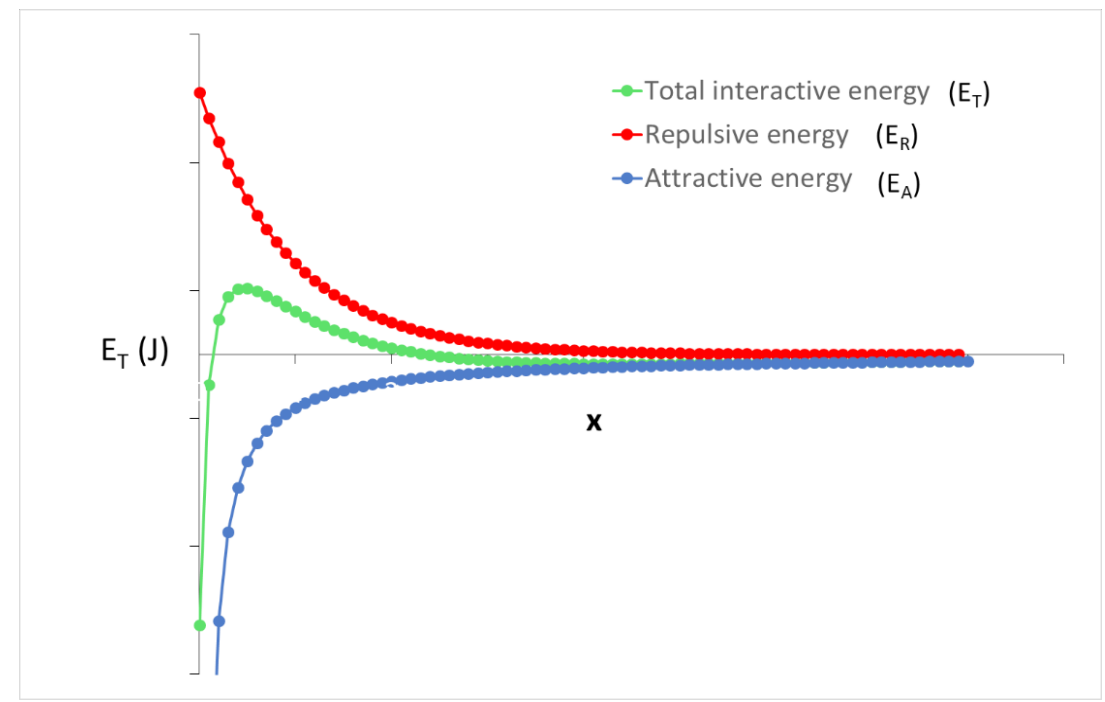

Figure 5. A typical profile of the interaction energies $\left(E_{A}, E_{R}, E_{T}\right)$ between a pair of charged particles $x$ distance apart.

\subsection{Simplified submodels for $E_{T}$ and $W$}

In terms of energy barrier between two spherical particles of sizes $\bar{s}$ and $\hat{s}$, Reerink and Overbreek ${ }^{[9]}$ derived an expression for the stability factor $(W)$. This expression is (Marchisio and Fox ${ }^{[5]}$ ):

$\mathrm{W}$

$$
W=\frac{2 k}{\bar{s}+\hat{s}} \exp \left[{ }^{E_{a g g}} / k_{B} T\right]
$$

where $E_{a g g}$ is the energy barrier for aggregation (i.e. the maximum value of the $E_{T}$ curve in Figure 5). A dimensional analysis of Equation (11) shows that $w(\bar{s}, \hat{s})$ is a dimensionless number. Hence, $2 k /(\bar{s}+\hat{s})$ should be dimensionless. However, considering that $1 / k$ has a dimension of $L, 2 k /(\bar{s}+\hat{s})$ has the dimension of $L^{-2}$. Thus, Equation (11) is incorrect. It is therefore necessary to develop a new equation, to replace Equation (11).

Further, Reerink and Overbreek ${ }^{[9]}$ reported an expression for $W$ simpler than Eq. (11). In doing this, they considered identical spherical particles where $\bar{s}=\hat{s}$ and employed the Taylor series expansion on another expression for $E_{T}$ (a simpler expression than Eq. (10)) about the value of $x$ corresponding to the energy barrier $E_{a g g}$. This value of $x$ is the thickness of the electric double layer i.e. $x=k^{-1}[23]$. The final expression is:

$$
\operatorname{In} W=-g_{1} \operatorname{InCe}+g_{2}
$$

where $C_{e}$ is the concentration of potential determining ions (these are the ion species that induce surface charge on the particles), while $g_{1}$ and $g_{2}$ are constants. The value of $g_{1}$ depends on the surface potential, while the value of $g_{2}$ depends on the physical system only ${ }^{\left[{ }^{[9]}\right.}$ They tested Equation (12) using a colloid of silver iodide particles in aqueous barium nitrate 
and then reported almost linear curves of $\operatorname{In} W$ vs $\operatorname{In} C_{e}$ thus confirming the linear relationship in Equation (12). Thereafter, Kumar et al. ${ }^{[10]}$ employed the expression in Equation (12) for $W$ in their model for the citrate synthesis method.

Here, in this review of the mathematical derivation of Reerink and Overbreek ${ }^{[9]}$ in comparison with Figure 5, we observe some inconsistencies, reported as follows.

First, Reerink and Overbreek ${ }^{[9]}$ did not start with Equations. (6) and (10). Instead of Equation (6) for $W$, they started with

$$
W=2 \int_{0}^{\infty} \exp \left(E_{T} / k T\right) \frac{d x_{s}}{\left(x_{s}+2\right)^{2}}
$$

where

$$
x_{s} \equiv \frac{2 D_{0}}{\bar{s}}
$$

where $D_{0}$ is the shortest distance between the two particles. Based on this definition, $D_{0}$ should be zero since the particles should be in contact when they aggregate. However, according to Reerink and Overbreek ${ }^{[9]}$, it is finite and nonzero. We think that $D_{0}$ should be $x$, the distance between the particles, not the shortest distance between them.

We observe three things: firstly, the two particles are equal in size $\overline{\boldsymbol{S}}$, thereby depicting monodispersed particles, but both particle sizes $\overline{\boldsymbol{s}}$ and $\hat{\boldsymbol{s}}$ should appear in the model. Secondly, the integration in Equation (13) is from $x_{s}=0$ to $x_{s}$ $=\infty$, but when derived from Equation (6) assuming $D_{0}=x$, the integration should be from $x_{s}=2$ to $x_{s}=\infty$. Thirdly, the denominator of the integral function is $\left(x_{\mathrm{s}}+2\right)$, but the denominator should be $x_{\mathrm{s}}$ when derived from Equation (6). Also, instead of Equation (10), Reerink and Overbreek ${ }^{[9]}$ started with

$$
E_{T}=-\frac{A_{H}}{12 x_{s}}+B_{r} \exp \left(-k_{s} x_{s}\right)
$$

where $A_{H}$ is the Hamaker constant,

$$
\begin{aligned}
& B_{r}=2.31 \times 10^{-6} \bar{s} \frac{\alpha^{2}}{z^{2}} \\
& k_{S}=k \bar{s} / 2 \\
& \alpha=\frac{\exp \left(|z| \mathrm{e} \Psi 0 / 2 k_{B} T\right)-1}{\exp \left(|z| \mathrm{e} \Psi 0 / 2 k_{B} T\right)+1}
\end{aligned}
$$

and $|z|$ is the magnitude of the potential determining ion.

Next, Reerink and Overbreek ${ }^{[9]}$ employed the Taylor series expansion on $E_{T}$ in Equation (13) about the value of $x_{s}$ corresponding to the energy barrier $E_{a g g}$, denoted as $x_{s m}$. As previously mentioned, this value corresponds to $x=k^{-1}$. Furthermore, they replaced the lower limit in the integral in Equation (13) by - $\infty$, claiming that this replacement introduces only a negligible error. Reerink and Overbreek ${ }^{[9]}$ differentiated Equation (15) with respect to $x_{s}$ and then equated the derivative to zero at $x_{s}=x_{s m}$. Again, Reerink and Overbreek ${ }^{[9]}$ considered a case where $E_{a g g}=0$. In the figure of Reerink and Overbreek ${ }^{[9]}$, which is similar to Figure 5, this case occurs when $E_{R}$ i.e. there is no electrostatic repulsion. As previously mentioned, Reerink and Overbreek ${ }^{[9]}$ employed this case to derive the expression in Equation (12), which was used in the model for the citrate synthesis as developed by Kumar et al. ${ }^{[10]}$ and discussed in Agunloye et al. ${ }^{[1]}$. By further rearrangements, Equation (13) becomes: 


$$
\operatorname{In} W=-\frac{A_{H}}{12 x_{\mathrm{sm}} k_{B} T} \operatorname{In} C_{e}-\frac{A_{H}}{12 x_{\mathrm{sm}} k_{B} T} \operatorname{In}\left(\frac{x_{s m} \bar{s}}{2 \sqrt{\epsilon_{0} \epsilon_{c} k_{B} T}}\right)+\operatorname{In}\left(\frac{\sqrt{\pi}}{\left(\frac{A_{H}}{24 x_{s m}{ }^{3} k_{B} T}\right)\left(2-k_{s} x_{s m}\right)^{1 / 2}} \frac{1}{\left(x_{s m}+2\right)^{2}}\right)
$$

Reerink and Overbreek ${ }^{[9]}$ assumed that apart from $C_{e}$, every other term in Equation (19) is a constant. Thus, they proposed that $W$ varies with $C_{e}$ according to Equation (12).

It is evident from Equation (19) that $W$ also varies with size $\bar{s}$. As previously shown, another limitation of this equation is that it assumes $E_{R}=0$, implying no stabilization by the repulsion energy. In spite of these limitations, Kumar et al. ${ }^{[10]}$ employed Equation (19) to derive an expression for the aggregation process in the citrate synthesis method. As shown by Agunloye et al, ${ }^{[11]}$, this expression fails to accurately describe the aggregation process.

\section{Discussion}

With the inconsistencies shown above, much errors would have been carried into the aggregation stabilization models developed by Reerink and Overbreek ${ }^{[9]}$ and employed by Kumar et al. ${ }^{[10]}$. These errors have been shown to have arisen from assumptions, property correlations and mathematical operations. The assumption that particles sizes are equal depicts aggregation of monodispersed particles. In the citrate synthesis method, this scenario would occur with the particle phase comprising gold atoms only as they have identical size. While this might be the reality shortly after the nucleation process, the model would, in particular, be unable to capture the evolution from gold atoms to the stable seed size in the particle phase, and in general, fail in describing the citrate synthesis method. Furthermore, the dimensional inconsistencies in the model shows a misplacement in properties and in their correlations, included during the model development process. For example, Equation (7) with the dimension of $L^{-2}$ would yield a value of unreasonable order of magnitude for the stability factor $(W)$. In Agunloye et al. ${ }^{[11]}$, extremely high values of $W$ were reported, thereby making aggregation insignificant in the citrate synthesis method.

The mathematical operation, when integration was done from from $x_{s}=0$ to $x_{s}=\infty$ in Equation (13), instead of from $x_{s}=2$ to $x_{s}=\infty$, would definitely introduce some error in the range of interaction distance being considered in the citrate synthesis method. GNPs produced in the synthesis have sizes 1-200 nm. The step change of 2 in the lower limit of $x_{s}$ would substantially impact calculations done at the nanoscale.

\section{Conclusions}

This paper presents a review of models for describing the aggregation process, which often indicates colloidal stability of nanoparticles, and corroborates them with experimental evidence from literature. The citrate synthesis method, a common route for producing the importantly employed spherical gold nanoparticles, was used to illustrate the aggregation process, thereby making a case for its modelling. Models from Fuchs ${ }^{[6]}$, describing the aggregation kernel by the Brownian motion, and those from DLVO, describing the electrostatic effect, were reviewed. The integrated model and its simplifications by Reerink and Overbreek ${ }^{[9]}$ were also discussed. This review highlights some inconsistencies in both the original model and the simplified form, thereby necessitating a new model for describing the aggregation process. 


\section{List of Symbols}

\begin{tabular}{|c|c|c|}
\hline Symbol & Meaning & Units \\
\hline \multicolumn{3}{|l|}{ Roman alphabets } \\
\hline$A_{H}$ & Hamaker constant & $J$ \\
\hline $\mathrm{Br}$ & Pre-exponential constant for repulsion energy & $J$ \\
\hline$C_{e}$ & Concentration of potential determining ions & $\mathrm{mol} / \mathrm{m}^{3}$ \\
\hline$D$ & Distance apart & $m$ \\
\hline$D_{0}$ & Shortest distance between the two particles & $m$ \\
\hline$D_{\overline{\mathrm{s}}}$ & Diffusion coefficient particles of size $\bar{s}$ & $m^{2} / s$ \\
\hline$D_{\hat{s}}$ & Diffusion coefficient particles of size $\hat{s}$ & $m^{2} / s$ \\
\hline$e$ & The charge on an electron, whose value & $C$ \\
\hline$E_{A}$ & Energy due to the Van der Waal's force of attraction & $J$ \\
\hline$E_{R}$ & Energy due to the charge repulsion & $J$ \\
\hline$E_{T}$ & $\begin{array}{l}\text { the sum of the particles' interaction energy due to the Van der } \\
\text { Waal's force of attraction and that due the charge repulsion }\end{array}$ & $J$ \\
\hline$E_{\text {agg }}$ & the energy barrier to particle aggregation & $J$ \\
\hline$E_{\max }$ & The maximum particles' interactive energy attainable & $J$ \\
\hline$E_{a}$ & Activation energy & $\mathrm{J} / \mathrm{mol}$ \\
\hline$F_{R}$ & Repulsive force & $N$ \\
\hline$F_{Z}$ & Stability gradient correlation parameter & - \\
\hline$k_{B}$ & The Boltzmann constant & $J / K$ \\
\hline$P_{r}$ & Osmotic pressure & $N / m^{2}$ \\
\hline$p_{i}$ & The number concentration of an ion in the bulk of the solution & $1 / m^{3}$ \\
\hline$q$ & centre to centre distance between the two particles & $m$ \\
\hline$Q$ & parameter that depends on the physical system & - \\
\hline$R$ & Universal gas constant & $8.31 \mathrm{~J} /(\mathrm{mol} . \mathrm{K})$ \\
\hline$s$ & Size & $m$ \\
\hline $\bar{s}$ & Size & $m$ \\
\hline$\hat{s}$ & Size & $m$ \\
\hline$t$ & Time & $s$ \\
\hline$t$ & Temperature & $K$ \\
\hline$V_{p 1}$ and $V_{p 2}$ & Particle volumes & $m^{3}$ \\
\hline$W$ & Stability factor & - \\
\hline$\tilde{\omega}_{A}(\bar{s}, \hat{s}, t)$ & Aggregation kernel & $\mathrm{m}^{3} / \mathrm{s}$ \\
\hline$x$ & distance between particles & $m$ \\
\hline$x_{s}$ & Transformed distance & - \\
\hline$x_{s m}$ & Transformed distance peak & - \\
\hline$z_{i}$ & The charge on the ion & $C$ \\
\hline \multicolumn{3}{|c|}{ Greek alphabets } \\
\hline$\epsilon_{0}$ & The permittivity of free space & $F / m$ \\
\hline$\epsilon_{c}$ & The dielectric constant of the solution & - \\
\hline$k$ & the Debye-Huckel parameter & $1 / m$ \\
\hline$k_{s}$ & Transformed $k$ & - \\
\hline$\mu$ & Fluid viscosity & $\mathrm{kg} /(\mathrm{m} . \mathrm{s})$ \\
\hline$\rho_{e}$ & Electron densities & $1 / m^{3}$ \\
\hline$\rho_{x}$ & counterion density profile & $1 / m^{3}$ \\
\hline$\Lambda$ & Chemical potential & $J$ \\
\hline$\varphi$ & Surface charge potential & $m V$ \\
\hline$\Psi_{x}$ & Electrostatic charge potential & $V$ \\
\hline
\end{tabular}

\section{References}

[1] Liveri, V. T. Controlled Synthesis of Nanoparticles in Microheterogeneous Systems, Nanostructure Science and Technology. Springer Science. 2006. https://link.springer.com/book/10.1007/b137569.

[2] Wuithschick, M., Witte, S., Kettemann, F., Rademann, K., Polte, J. Turkevich in New Robes: Key Questions Answered for the Most Common Gold Nanoparticle Synthesis. Phys. Chem. 2015; 17: 19895-19900. doi: 10.1021/ acsnano. 5 b01579s. 
[3] Agunloye, E., Panariello, L., Gavriilidis, A., Mazzei, L. A Model for the Formation of Gold Nanoparticles in the Citrate Method. Chemical Engineering Science. 2018; 173: 275-286. doi:10.1016/j.ces.2018.06.046.

[4] Ramkrishna, D. Population Balances. Academic Press. 2000. https://www.elsevier.com/books/population-balances/ ramkrishna/978-0-12-576970-9.

[5] Marchisio, D. L., Fox, R. O. Computational Models for Polydisperse Particulate and Multiphase Systems. Cambridge University Press. 2013. https://doi.org/10.1017/CBO9781139016599.

[6] Fuchs, N. A. The Mechanics of Aerosols. New York, Pergamon; 1964. https://doi.org/10.1002/qj.49709138822.

[7] Derjaguin, B., and Landau, L. Theory of the stability of strongly charged lyophobic sols and of the adhesion of strongly charged particles in solutions of electrolytes. Prog. Surf. Sci. 1941; 43: 30-59. doi: 10.1016/0079-6816(93)90013-L.

[8] Verwey, E. J. W., Overbeek, J. T. G. Theory of the Stability of Lyophobic Colloids. Amsterdam: Elsevier; 1948. https:// doi.org/10.1021/j150453a001.

[9] Reerink, H., Overbeek, J. T. G. The rate of coagulation as a measure of the stability of silver iodide sols. Discuss. Faraday Soc. 1954; 18: 74-84. doi: 10.1039/DF9541800074.

[10] Kumar, S., Kumar, R., Gandhi, K. S., Modeling of Formation of Gold Nanoparticles by Citrate Method. Ind. Eng. Chem. Res. 2007; 46: 3128-3136. doi: 10.1021/ie060672j

[11] Agunloye, E., Gavriilidis, A., Mazzei, L. A Mathematical Investigation of the Turkevich Organizer Theory in the Citrate Method for the Synthesis of Gold Nanoparticles. Chemical Engineering Science. 2017. http://dx.doi. org/10.1016/j.ces.2017.07.032.

[12] Chow, M., Zukoski, C. Gold Sol Formation Mechanisms: Role of Colloidal Stability. J. Colloid Interface Sci. 1994; 165: 97-109. https://doi.org/10.1006/jcis.1994.1210.

[13] Hauser, E., A., Lynn, J., E. Experiments in Colloid Chemistry. McGraw-Hill, New York; 1940. https://doi.org/10.1021/ ed018p349.4.

[14] Turkevich, J., Stevenson, P., Hillier, J. A Study of the Nucleation and Growth Process in the Synthesis of Colloidal Gold. Discuss. Faraday Soc. 1951; 11: 55. https://www.scienceopen.com/document?vid=c897f2d6-743a-406d-8072bc56532d7961.

[15] Ji, X. H., Song, X. N., Li, J., Bai, Y. B., Yang, W. S., Peng, X. G. Size Control of Gold Nanocrystals in Citrate Reduction: The Third Role of Citrate, J. Am. Chem. Soc. 2007; 129: 13939. doi:10.1021/ja074447k.

[16] Polte, J., Ahner, T. T., Delissen, F., Sokolov, S., Emmerling, F.,Thünemann, A. F., Kraehnert, R. Mechanism of Gold Nanoparticle Formation in the Classical Citrate Synthesis Method Derived from Coupled In Situ XANES and SAXS Evaluation. J. Am. Chem. Soc. 2010; 132: 1296-1301. https://doi.org/10.1021/ja906506j.

[17] Cushing, B. L., Kolesnichenko, V. L., Connor, C. J. O. Recent Advances in the Liquid-Phase Syntheses of Inorganic Nanoparticles. Chem. ReV. 2004; 104: 3893. http://www.eng.uc.edu/ beaucag/Classes/Nanopowders/ OConnorCJReviewofNanoSynthesis.pdf.[3] Bogush, G.H., Zukoski IV, C.F. Uniform silica particle precipitation: An aggregative growth model. J. Colloid Interface Sci. 1991; 142: 19-34. doi:10.1016/0021-9797(91)90030-C.

[18] Brust, M., Walker, M., Bethell, D., Schiffrin, D. J.,Whyman, R. Synthesis of Thiol-Derivatised Gold Nanoparticles in a Two-Phase Liquid-Liquid System. Chem. Commun. 1994; 801. doi:10.1039/C39940000801.

[19] Smoluchowski, M. v. Mathematical Theory of the Kinetics of the Coagulation of Colloidal Solutions. Z. Phys. Chem. 1917; 92: 129-68. https://apps.dtic.mil/dtic/tr/fulltext/u2/841609.pdf.

[20] Hunter, R. Foundations of colloid science. Oxford, U.K; Clarendon Press. 1995. pp 441-447. https://www.amazon. com/Foundations-Colloid-Science-Robert-Hunter/dp/0198505027.

[21] Bogush, G.H., Zukoski IV, C.F. Uniform silica particle precipitation: An aggregative growth model. J. Colloid Interface Sci. 1991; 142: 19-34. doi:10.1016/0021-9797(91)90030-C.

[22] Polte J. Fundamental growth principles of colloidal metal nanoparticles-a new perspective. CrystEngComm 2015; 17(36): 6809-6830. doi: 10.1039/C5CE01014D.

[23] Israelachvili, J. N. Intermolecular and surface forces, $3^{\text {rd }}$ edition. Burlinton, MA; Academic Press. 2011. https://www. elsevier.com/books/intermolecular-and-surface-forces/israelachvili/978-0-12-391927-4. 